\title{
The Asiago Database of Spectroscopic Databases (ADSD) $)^{\star} \star \star$
}

\author{
R. Sordo ${ }^{1,2}$ and U. Munari ${ }^{1}$ \\ 1 INAF Osservatorio Astronomico di Padova, via dell'Osservatorio 8, 36012 Asiago (VI), Italy \\ e-mail: munari@pd.astro.it \\ 2 Dipartimento di Astronomia, Univ. di Padova, vicolo della Specola 5, 35122 Padova, Italy
}

Received 1 December 2005 / Accepted 20 February 2006

\begin{abstract}
Databases of observed stellar spectra are continuously being published and made publicly available, and the average number of stars per database is increasing. This paper reviews the current status. The Asiago Database of Spectroscopic Databases (ADSD) aims to provide a census of publicly available libraries of observed stellar spectra, to document their content and to homogenize their parameters for easier consultation and access. Refereed journals, conference proceedings and personal web pages have been searched for libraries of a given minimum size, properly documented and with data made publicly and directly accessible. A total of 294 databases ( 54 ultraviolet, 183 optical, 50 infrared and 7 combined) have been found to match the selection criteria and have been included in ADSD. They provide spectra of 16046 different stars in electronic or printed formats. A card for each library describes in a homogeneous way its aims, content, type of data, caveats, data download links, source paper, properties of included stars and more. A dedicated web page allows direct access to ADSD, plans future updates, and provides interrogation tools to search all the libraries matching given characteristics or including any given star.
\end{abstract}

Key words. astronomical data bases: miscellaneous - catalogs - surveys - atlases - stars: general

\section{Introduction}

The production of libraries of observed stellar spectra has been increasing in recent decades, with both an increase in the number of datasets and in the number of stars included. This is arguably the result of an increasing observational efficiency (from spectral scanners, to single array Reticon, to CCD, to multiple object spectroscopy via fiber feeding), of a larger amount of observational time being allocated to survey projects on medium size telescopes, and of the increasing use by astronomers of electronic media to support and publicize their research work.

A census, a parameter homogenization and a web interrogation tool of libraries of observed stellar spectra are needed. To this aim we have created the Asiago Database on Spectroscopic Databases (ADSD). Our effort aims to prevent (unnecessary) duplication of observational efforts, increase the awareness of the enormous wealth of already existing data and information, stimulate a critical review and comparison of their content, provide a guided access to them and speed up the identification and selection of data of interest. Early efforts on this project were described by Sordo \& Munari (2003), while some statistical applications were presented by Munari \& Sordo (2005). We have surveyed refereed journals, conference proceedings, personal web pages, CDS/ADC on-line catalog repositories and astro-ph documents to search for existing libraries of optical, ultraviolet and infrared spectra of stars. We are confident that the large majority of existing libraries have been located and included in ADSD. The effort is updated to the time of manuscript submission. At the time of writing the ADSD includes 294 spectroscopic

* Tables 2-4 are only available in electronic form at http://www. edpsciences.org

$\star \star$ A printable book-size version of ADSD can be downloaded from the project web site http://web.pd.astro.it/adsd/index.html
Table 1. How the 294 libraries censused in ADSD distribute between optical, ultraviolet and infrared ranges and electronic, tabular or graphical data type.

range $\quad \lambda \lambda$ interval total $n$. electronic printed graphical
libraries

$\begin{array}{lcrrrr}\text { Ultraviolet } & 70-3000 \AA & 54 & 32 & 12 & 10 \\ \text { Optical } & 3000-10000 \AA & 183 & 85 & 52 & 46 \\ \text { Infrared } & 1-25 \mu \mathrm{m} & 50 & 21 & 1 & 28 \\ \text { Combined } & & 7 & 6 & & 1\end{array}$

databases. Their subdivision into optical, ultraviolet and infrared branches and electronic, printed and graphical types is outlined in Table 1. The total number of stars whose spectrum is included in the electronic or printed databases (thus excluding those providing data in graphical form only) is 16046 . It is divided into 9405 different stars included in optical libraries, 3184 in UV libraries and 6095 in IR libraries. Tables $2-4$ provide a list of the 294 libraries censused in ADSD.

\section{Full size ADSD web edition}

The ADSD is composed of a printable book-size version and by a web portal providing browsing facilities and search/interrogation tools described below.

The printable book-size version is too long ( 400 pages) for formal publication here, and it is instead offered for download from the project website: http://web.pd.astro.it/ adsd/index .html. Full details and explanatory notes are provided there. 
Valdes et al. (2004)

The Indo-US spectral library of medium resolution spectra, covering the whole HR diagram and aiming to support automated spectral classification and galaxy population synthesis. The stellar radial velocities have been compensated for and the telluric absorptions divided out. The shape of the continumm is fixed by imposing the match with the corresponding energy distribution for the stor's spectral type from mposing the match with the corresponding energy distribution for the star's spectral type

ref. paper: Valdes, F., Gupta, R., Rose, J. A., Singh, H. P. \& Bell, D. J., 2004, ApJS 152, 251
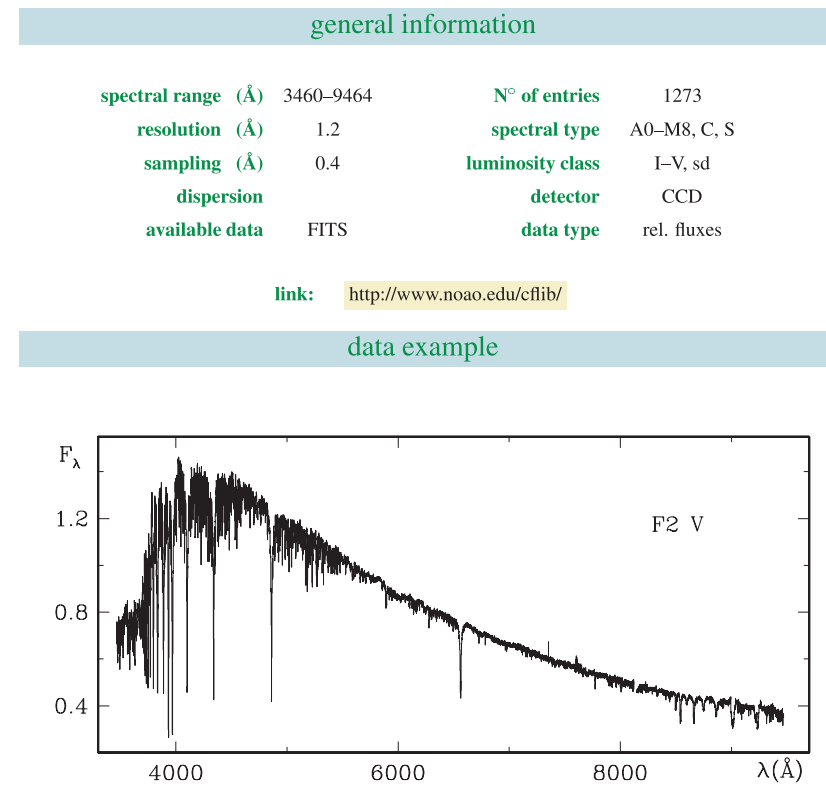

spectral type coverage
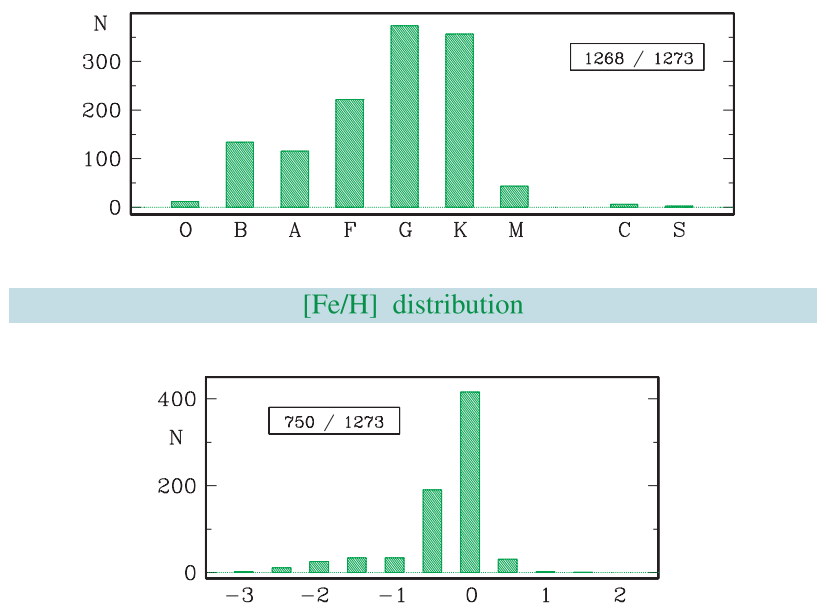

luminosity class distribution

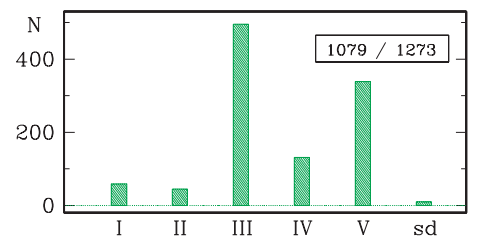

Fig. 1. An example of the card accompanying each individual database censused in ADSD, showing its front and rear sides. The front one describes the content of the library and presents an example of a spectrum. The rear one uses instead information retrieved from SIMBAD to illustrate the distribution in spectral type, luminosity class and metallicity of the stars contained in the database. A detailed description of the content of each entry and coding syntax is provided in the full book-size printable version of ADSD paper that can be downloaded from the project web site.

Future updates of the ADSD live edition are planned and will be made available via the web portal.

\section{Census boundaries}

The spectroscopic databases included in ADSD are divided into three broad categories: electronic are the libraries for which the spectra can be accessed and retrieved in electronic form, from an ftp or web address; printed are the libraries for which the spectra are available only in the form of printed tables with no known transfer to an electronic version; graphical are the libraries presenting their spectra only as figures, with no known transfer to a printed table or electronic version.

To be included in the ADSD a spectroscopic database $(i)$ must be accompanied by a publicly available publication that documents the data (preferably in a refereed journal), (ii) the data must be directly accessible, thus excluding the datasets that need to be requested from and/or negotiated directly with the Authors, and (iii) the library must contain spectra of $\sim 10$ stars minimum. Notable exceptions are the libraries devoted to specific types of objects (like brown dwarfs, Be stars in clusters, or WR stars in LMC., etc.), or very high resolution reference spectral atlases devoted to single stars (like Arcturus; ADSD however does not include atlases of the Sun), or compilation of spectral energy distributions of key reference spectrophotometric standards. There are a few exceptions to the above criteria, depending on our judgment of the relevance of a given atlas, driven not only by the number of stars and their peculiarity/rarity/specificity but also on the quality of the data, their wavelength extension, type of sampling and citation frequency in the literature.

ADSD does not include general repositories of unselected data collected by ground based telescopes or space missions (like HST). For example, the INES ${ }^{1}$ archive of all spectra secured by the IUE satellite (maintained at Goddard, VILSPA and several national mirrors) is not included in ADSD, while the Jamar et al. (1976) atlas of selected TD-1 ultraviolet spectra of 1356 bright stars is included. The IRAS atlas of 5425 lowresolution spectra is included in ADSD because the spectra were all individually plotted and discussed in a monographic issue of A\&AS and poor spectra have been removed. ADSD also does not include the unpublished sets of spectra about internal reference and calibration stars.

\section{ADSD structure}

\section{1. $A D S D$ cards and comparative tables}

For each spectroscopic database providing data in either electronic or printed formats, there is an individual ADSD card describing its main characteristics and content. An example is presented in Fig. 1. Correspondence between card numbers and libraries is given in the first column of Tables 2 and 3. Spectroscopic databases presenting data only in graphical form do not have their own cards. 
The cards aim to facilitate the inter-comparison between the different spectroscopic databases, by providing the same type of information, homogenized and accompanied by a sample spectrum representative of those included in the library. The content of the cards and their symbol syntax are described in detail in the printable book-size version of ADSD available from the project web site.

Tables 2-4 provide a summary of the 294 libraries currently contained in ADSD, allowing an easy comparison of their basic characteristics for an easier location of those of interest.

\subsection{The web portal and interrogation tools}

The ADSD web portal provides interrogation tools that allows one to search the list of censused databases for a number of possible applications. Two examples of a query illustrate the possibilities.

In the first type of interrogation, ADSD is searched for the spectral databases that meet certain parameters, e.g. ADSD could be searched for the libraries that satisfy all of the following criteria at the same time: $(a)$ they must include the 4000-5000 A range, (b) at a resolution between 1 and $10 \AA$, $(c)$ with data calibrated into absolute fluxes, that are $(d)$ accessible in electronic form, and $(e)$ that contain G2 III spectra of individual stars, not mean spectra. In such a case, the web interrogation tool would answer that the only two catalogs matching the requirements are (i) Jacoby et al. (1984) presenting spectra of two G2 III stars HD 28099 and HD 66171, and (ii) Jones (1996, included in Leitherer 1996) providing spectra of the three G2 III stars HD 4307, HD 76151 and HD 98553.
In the second type of interrogation, ADSD is asked to list all libraries that contain spectra (in electronic or tabular form) of a given star. The star name must be introduced in a format accepted by SIMBAD (examples are given), that provides the list of other names recognized for this star and that are used to search all censused libraries. Asking for example for star HD 99028 (=HR 4399) would give in return Jones (1996, included in Leitherer 1996), Kharitonov et al. (1988), Heck et al. (1984), Breger (1976), Burnashev (1985) and Dickens \& Penny (1971).

Acknowledgements. MIUR COFIN2001 and INAF Osservatorio Astronomico di Padova provided funding support to R.S. We would like to thank R.Barbon for his kind support of the project, that was initiated by Federico Boschi during his laurea dissertation on Gaia spectroscopy in 1999.

\section{References}

Breger, M. 1976, ApJS, 32, 7

Burnashev, V. I. 1985, AbaOB, 59, 83

Dickens, R. F., \& Penny, A. F. 1971, MNRAS, 153, 287

Heck, A., Egret, D., Jaschek, M., Jaschek, C., et al. 1984, A\&AS, 57, 213

Jacoby, G. H., Hunter, D. A., \& Christian, C. A. 1984, ApJS, 56, 257

Jamar, C., et al. 1976, ESA SR-27

Kharitonov, A. V., Tereshchenko, V. M., \& Knjazeva, L. N. 1988, The spectrophotometric catalogue of stars of Alma-Ata Observatory (Nauka Pub)

Leitherer, C., Alloin, D., Fritze-v. Alvensleben, U., et al. 1996, PASP, 108, 996

Munari, U., \& Sordo, R. 2005, in ATLAS12 and Related Codes, MemSAIt Suppl., 8,170

Sordo, R., \& Munari, U. 2003, in Gaia Spectroscopy, Science and Technology, ASPC Conf. Ser., 298, 221 
R. Sordo and U. Munari: The Asiago Database of Spectroscopic Databases (ADSD), Online Material p 1

\section{Online Material}


R. Sordo and U. Munari: The Asiago Database of Spectroscopic Databases (ADSD), Online Material p 2

Table 2. The list of optical, ultraviolet, infrared and combined libraries that give access to the spectra in electronic form and that are included in ADSD. The first column lists the number of the figure on the ADSD web site that provide access to the card of the given library and that is included in the printable book-size full version of ADSD.

\begin{tabular}{c|c|c|c|c|c|c|c|c}
\hline $\begin{array}{c}\text { Fig } \\
\text { N. }\end{array}$ & $\begin{array}{c}\text { Authors and year } \\
\text { of publication }\end{array}$ & $\begin{array}{c}\text { Spectral } \\
\text { Range } \\
(\AA)\end{array}$ & $\begin{array}{c}\text { D } \\
(\AA / p i x) \\
(\AA / m m)\end{array}$ & $\begin{array}{c}\text { R or } R_{P} \\
(\AA)\end{array}$ & $\begin{array}{c}\text { Samplig } \\
(\AA)\end{array}$ & $\begin{array}{c}\text { N. } \\
\text { Stars }\end{array}$ & $\begin{array}{c}\text { Spectrum } \\
\text { Lum. } \\
\text { Class }\end{array}$ & $\begin{array}{c}\text { detector/ } \\
\text { instr. }\end{array}$ \\
\hline
\end{tabular}

Optical libraries with data in electronic form

\begin{tabular}{|c|c|c|c|c|c|c|c|c|c|c|c|}
\hline 6 & Adelman et al. & 1989 & $3300-10800$ & & $4-50$ & & 207 & O9-K4, pec & $\mathrm{I}-\mathrm{V}$ & Sp.Sc. & rel \\
\hline 7 & Alekseeva et al. & 1997 & $\begin{array}{l}3200-7350 \\
3200-10800\end{array}$ & & $\begin{array}{c}50 \\
100 \\
\end{array}$ & $\begin{array}{l}25 \\
25\end{array}$ & $\begin{array}{l}602 \\
278 \\
\end{array}$ & $\begin{array}{l}\text { O5-M4 } \\
\text { O5-M4 }\end{array}$ & $\begin{array}{l}\mathrm{I}-\mathrm{V} \\
\mathrm{I}-\mathrm{V}\end{array}$ & $\begin{array}{l}\text { Sp.Sc. } \\
\text { Sp.Sc. }\end{array}$ & $\begin{array}{l}\text { abs } \\
\text { abs }\end{array}$ \\
\hline 8 & Allen \& Strom & 1995 & 5600-9600 & & 6 & 1.97 & 102 & F1-M4 & $\mathrm{V}$ & $\mathrm{CCD}$ & counts \\
\hline 9 & Allende Prieto et al. & 2004 & $\begin{array}{l}3620-10440 \\
3620-9210 \\
\end{array}$ & & $\begin{array}{l}50000 \\
45000 \\
\end{array}$ & $\begin{array}{l}0.01 \\
0.01 \\
\end{array}$ & $\begin{array}{l}91 \\
28 \\
\end{array}$ & $\begin{array}{l}\text { A0-K3 } \\
\text { F2-M0 }\end{array}$ & $\begin{array}{l}\text { II-V } \\
\text { III-V }\end{array}$ & $\begin{array}{l}\mathrm{CCD} \\
\mathrm{CCD}\end{array}$ & $\begin{array}{l}\text { norm } \\
\text { norm }\end{array}$ \\
\hline 10 & Andrillat et al. & 1995 & $8375-8770$ & 33 & $1-1.5$ & 0.8 & 76 & O5-G0, pec & $\mathrm{I}-\mathrm{V}$ & $\mathrm{CCD}$ & norm \\
\hline 11 & Appelquist et al. & 1983 & $5185-8700$ & 4.9 & 0.195 & 0.04 & 1 & $\gamma$ Tau (K0) & III & plate & norm \\
\hline 12 & Bagnulo et al. & 2003 & $3040-10400$ & & 80000 & $0.012 \div 0.026$ & 449 & $\mathrm{O}-\mathrm{M}, \mathrm{C}$ & $\mathrm{I}-\mathrm{V}, \mathrm{sd}$ & $\mathrm{CCD}$ & rel \\
\hline 13 & Barnbaum & 1994 & $5080-7850$ & $2.84-4.35$ & 47000 & $0.043 \div 0.065$ & 89 & $\mathrm{C}$ & & $\mathrm{CCD}$ & counts \\
\hline 14 & Barnbaum et al. & 1996 & $4000-7000$ & $0.85-3.25$ & $1.6-6.5$ & $0.85 \div 3.25$ & 72 & $\mathrm{C}, \mathrm{Ba}, \mathrm{CH}$ & & several & counts \\
\hline 15 & Biryukov et al. & 1998 & $3425-7525$ & & 50 & 50 & 82 & $\mathrm{~B} 8-\mathrm{K} 1$ & III-V & Sp.Sc. & abs \\
\hline 16 & Breger & 1976 & $3200-12000$ & & $10-100$ & 50-200 & 609 & $\mathrm{O} 5-\mathrm{K} 7$ & $\mathrm{I}-\mathrm{V}$ & Sp.Sc. & rel \\
\hline 17 & Burgasser et al. & $2003 \mathrm{a}$ & 6300-10100 & 1.86 & 7 & 1.86 & 13 & $\mathrm{~T}$ & $\mathrm{~V}$ & $\mathrm{CCD}$ & abs \\
\hline 18 & Burnashev & 1985 & $3200-7350$ & & 25 & 25 & 1562 & O5-M7,WR,C,S,pec & $\mathrm{I}-\mathrm{V}$ & & abs \\
\hline 19 & Carquillat et al. & 1997 & $8380-8780$ & 33 & 2 & 0.8 & 54 & $\mathrm{~A} 2-\mathrm{M} 4, \mathrm{C}, \mathrm{S}$ & $\mathrm{I}-\mathrm{V}, \mathrm{sd}$ & $\mathrm{CCD}$ & norm \\
\hline 20 & Castelli \& Hubrig & 2004 & $3040-10000$ & & 90000,110000 & $0.016 \div 0.026$ & 1 & HD 175640 (B9) & $\mathrm{V}$ & $\mathrm{CCD}$ & norm \\
\hline 21 & Cenarro et al. & 2001 & $8348-9020$ & $0.79-0.85$ & $1.5-2.13$ & 0.85 & 706 & O6-M8 & $\mathrm{I}-\mathrm{V}, \mathrm{sd}$ & $\mathrm{CCD}$ & rel \\
\hline 22 & Cincunegui \& Mauas & 2004 & $\begin{array}{l}3890-6650 \\
3885-6975 \\
\end{array}$ & $\begin{array}{c}0.141 \div 0.249 \\
3.41\end{array}$ & $\begin{array}{c}26400 \\
1050 \div 2070\end{array}$ & $\begin{array}{c}0.149 \\
3.41 \\
\end{array}$ & $\begin{array}{l}91 \\
91 \\
\end{array}$ & $\begin{array}{l}\text { F6-M5.5 } \\
\text { F6-M5.5 }\end{array}$ & $\begin{array}{l}\text { IV-V } \\
\text { IV-V }\end{array}$ & $\begin{array}{l}\mathrm{CCD} \\
\mathrm{CCD} \\
\end{array}$ & $\begin{array}{l}\text { abs } \\
\text { abs }\end{array}$ \\
\hline 23 & Clampitt \& Burstein & 1997 & $3500-7780$ & & $32-64$ & $65-680$ & 237 & $\mathrm{~B}-\mathrm{K} 0$, pec & III-V & Sp.Sc. & rel \\
\hline 24 & Danks \& Dennefeld & 1994 & $5800-10200$ & 4.3 & & 4 & 137 & O5-M7, pec & $\mathrm{I}, \mathrm{III}, \mathrm{V}$ & Reticon & abs \\
\hline 25 & Davies et al. & 2005 & 5846-7030 & & $1.1-2.1$ & 0.447 & 13 & B0-A6 & Ia, Iae & $\mathrm{CCD}$ & norm \\
\hline 26 & Fluks et al. & 1994 & $3500-10000$ & & $1300 \div 9000$ & 1 & $22 \mathrm{~ms}$ & M0-M10 & III & $\mathrm{CCD}, \mathrm{IDS}$ & abs \\
\hline 27 & Friedrich et al. & 2000 & $3200-9200$ & $100 \div 500$ & $5-40$ & $2.4-7.6$ & 40 & WD & & $\mathrm{CCD}$ & abs \\
\hline 28 & Glushneva et al. & 1992 & $3225-7675$ & & 50 & 50 & 238 & O7-M4, pec & $\mathrm{I}-\mathrm{V}$ & Sp.Sc. & abs \\
\hline 29 & Glushneva et al. & $1998 \mathrm{a}$ & $5975-10825$ & & 50 & 50 & 223 & O9-M5, pec & $\mathrm{I}-\mathrm{V}$ & Sp.Sc. & abs \\
\hline 30 & Glushneva et al. & $1998 b$ & $3225-7625$ & & 50 & 50 & 862 & O6-M6, pec & $\mathrm{I}-\mathrm{V}$ & Sp.Sc. & abs \\
\hline 31 & Gray et al. & 2003 & $\begin{array}{l}3800-4600 \\
3800-5500 \\
\end{array}$ & & $\begin{array}{l}1.8,3.6 \\
1.8-3.6 \\
\end{array}$ & $\begin{array}{l}1.11-1.14 \\
0.77-1.79 \\
\end{array}$ & $\begin{array}{r}3413 \\
215 \\
\end{array}$ & $\begin{array}{l}\text { B3-M4, WR } \\
\text { O4-M7, pec }\end{array}$ & $\begin{array}{l}\mathrm{I}-\mathrm{V}, \mathrm{sd} \\
\mathrm{I}-\mathrm{V}\end{array}$ & $\begin{array}{l}\mathrm{CCD} \\
\mathrm{CCD} \\
\end{array}$ & $\begin{array}{c}\text { all } \\
\text { norm }\end{array}$ \\
\hline 32 & Griffin \& Griffin & 1979 & $3140-7470$ & & & 0.005 & 1 & $\alpha$ CMi (F5) & IV-V & plate & norm \\
\hline 33 & Gunn \& Stryker & 1983 & $3130-10800$ & & 20,40 & 10,20 & 175 & O5-M8, pec & $\mathrm{I}-\mathrm{V}$ & Sp.Sc. & rel \\
\hline 34 & Hamann et al. & 1995 & $3300-9000$ & & $2000-3600$ & $1.12-3$ & 62 & $\mathrm{WN}$ & & $\mathrm{CCD}$ & norm \\
\hline 35 & Hamuy et al. & 1992 & $3300-7550$ & & 10,16 & 16,50 & 29 & B1-G0, WD, pec & III-V, sd & $\mathrm{CCD}$ & abs \\
\hline 36 & Hamuy et al. & 1994 & $6000-10500$ & & 11,16 & 16,50 & 30 & B1-G0, WD, pec & III-V, sd & $\mathrm{CCD}$ & abs \\
\hline 37 & Ivison et al. & 1994 & $4500-6800$ & 79 & & 2.25 & 32 & Symb. & & IPCS & abs \\
\hline 38 & Jacoby et al. & 1984 & $3510-7427$ & & 4.5 & 1.4 & 161 & O5-M7, pec & $\mathrm{I}-\mathrm{V}$ & Reticon & abs \\
\hline 39 & Johnson & 1977 & $4000-10000$ & & $1.9 \div 3.85$ & $3.855 \mathrm{~cm}^{-1}$ & 16 & O6-M9, C, S, pec & $\mathrm{I}-\mathrm{V}$ & FTS & ps.norm \\
\hline 40 & Johnson & $1978 \mathrm{a}$ & $4000-11000$ & & $1.9 \div 3.85$ & $3.855 \mathrm{~cm}^{-1}$ & 32 & O9.5-M2, C, S, pec & $\mathrm{I}-\mathrm{V}$ & FTS & ps.norm \\
\hline 41 & Johnson & 1980 & $4045-10220$ & & & $50-150$ & 16 & $\mathrm{O} 9-\mathrm{A} 3$ & III-V & FTS & abs \\
\hline 42 & Jones & 1996 & $3820 \cdots 5460$ & & 1.8 & 0.6 & 684 & O9-M7.5, C, pec & $\mathrm{I}-\mathrm{V}, \mathrm{sd}$ & CCD & abs \\
\hline 43 & Kharitonov et al. & 1988 & $3225-7575$ & & & 50 & 1147 & O6-M5, S, pec & $\mathrm{I}-\mathrm{V}$ & Sp.Sc. & abs \\
\hline 44 & Kiehling & 1987 & $3200-8600$ & & 10 & 10 & 60 & A9-M5 & $\mathrm{I}-\mathrm{V}$ & Sp.Sc. & rel \\
\hline 45 & Kirkpatrick et al. & 1999 & $6300-10100$ & & 9 & 1.865 & 25 & L0-L8 & $\mathrm{V}$ & $\mathrm{CCD}$ & rel \\
\hline 46 & Kirkpatrick et al. & 2000 & $6300-10100$ & & 9 & 1.9 & 67 & L0-L8 & $\mathrm{V}$ & $\mathrm{CCD}$ & rel \\
\hline 47 & Knyazeva \& Kharitonov & 1996 & $3225-7575$ & & & 50 & $41 \mathrm{~ms}$ & B5-M5 & III-V & Sp.Sc. & rel \\
\hline 48 & Le Borgne et al. & 2003 & $3200-9500$ & & 3 & 1 & 253 & O5-M6, WR, WD & $\mathrm{I}-\mathrm{V}, \mathrm{sd}$ & $\mathrm{CCD}$ & abs \\
\hline 49 & Malyuto et al. & 1997 & $\begin{array}{l}4800-7700 \\
4800-7700 \\
\end{array}$ & & $\begin{array}{l}10 \\
10 \\
\end{array}$ & $\begin{array}{l}5.7 \div 6.1 \\
5.7 \div 6.1\end{array}$ & $\begin{array}{l}15 \\
18 \\
\end{array}$ & $\begin{array}{l}\text { K0-M5 } \\
\text { M1-M4.5 }\end{array}$ & $\begin{array}{l}\text { I-III } \\
\text { I } \\
\end{array}$ & $\begin{array}{l}\text { Digicon } \\
\text { Digicon }\end{array}$ & $\begin{array}{l}\text { rel } \\
\text { abs }\end{array}$ \\
\hline 50 & Marrese et al. & 2003 & $8490-8740$ & & 20000 & 0.25 & 92 & F2-M7 & $\mathrm{I}-\mathrm{V}$ & $\mathrm{CCD}$ & norm \\
\hline 51 & Massey et al. & 1988 & $3110-8380$ & & $7-14$ & $1.6-2.7$ & 25 & $\mathrm{O}-\mathrm{A}, \mathrm{DA}, \mathrm{pec}$ & $\mathrm{I}-\mathrm{V}, \mathrm{sd}$ & IRS, IIDS & abs \\
\hline 52 & Meliani et al. & 1995 & $4250-5400$ & 114 & 5 & 1 & 40 & K5-M & I & IDS & rel \\
\hline
\end{tabular}


R. Sordo and U. Munari: The Asiago Database of Spectroscopic Databases (ADSD), Online Material p 3

Table 2. (continued)

\begin{tabular}{|c|c|c|c|c|c|c|c|c|c|c|c|}
\hline $\begin{array}{l}\text { Fig } \\
\text { N. }\end{array}$ & $\begin{array}{c}\text { Authors and year } \\
\text { of publication }\end{array}$ & & $\begin{array}{c}\text { Spectral } \\
\text { Range } \\
(\AA)\end{array}$ & 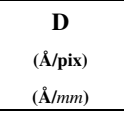 & $\begin{array}{c}\mathbf{R} \text { or } R_{P} \\
\quad \text { (A) }\end{array}$ & $\begin{array}{c}\text { Samplig } \\
\text { (A) }\end{array}$ & $\begin{array}{c}\text { N. } \\
\text { Stars }\end{array}$ & Spectrum & $\begin{array}{l}\text { Lum. } \\
\text { Class }\end{array}$ & $\begin{array}{l}\text { detector/ } \\
\text { instr. }\end{array}$ & data \\
\hline 53 & Mennickent \& Sterken & 1997 & $6470-8780$ & & 5.4 & 2.4 & 8 & $\mathrm{Be}$ & & $\mathrm{CCD}$ & norm \\
\hline 54 & Montes et al. & 1997 & $3830 \cdots 7643$ & & $0.2-3.1$ & $0.1-0.3$ & 115 & F0-M9 & II-V & $\mathrm{CCD}$ & norm \\
\hline 55 & Montes \& Martin & 1998 & $4800 \cdots 10600$ & & 55000 & $0.05 \div 0.09$ & 48 & F5-M8 & III-V & $\mathrm{CCD}$ & counts \\
\hline 56 & Montes et al. & 1999 & $3900 \cdots 9000$ & $0.07-0.38$ & 12000 & $0.15-0.35$ & 132 & F0-M8 & $\mathrm{I}-\mathrm{V}$ & $\mathrm{CCD}$ & counts \\
\hline 57 & Munari et al. & 1997 & $\begin{array}{l}4020-7890 \\
3480-7530 \\
\end{array}$ & & $\begin{array}{l}12 \\
20 \\
\end{array}$ & $\begin{array}{c}3.2 \div 4.0 \\
7.5-8 \\
\end{array}$ & $\begin{array}{l}14 \\
14 \\
\end{array}$ & $\begin{array}{l}\mathrm{CV} \\
\mathrm{CV}\end{array}$ & & $\begin{array}{l}\mathrm{CCD} \\
\mathrm{CCD} \\
\end{array}$ & $\begin{array}{l}\text { abs } \\
\text { abs }\end{array}$ \\
\hline 58 & Munari & 2003 & $8490-8740$ & & 20000 & 0.25 & 20 & peculiar & & $\mathrm{CCD}$ & norm \\
\hline 59 & Munari \& Tomasella & 1999 & $8490-8750$ & & 20000 & 0.25 & 130 & O4-M8 & $\mathrm{I}-\mathrm{V}$ & $\mathrm{CCD}$ & norm \\
\hline 60 & Munari \& Zwitter & 1998 & $3300-9100$ & & 7 & 2.9 & 20 & $\mathrm{CV}$ & & $\mathrm{CCD}$ & abs \\
\hline 61 & Munari \& Zwitter & 2002 & $\begin{array}{l}3210-9190 \\
3150-7530 \\
\end{array}$ & $\begin{array}{l}2.5 \\
7.5 \\
\end{array}$ & $\begin{array}{c}5 \\
15 \\
\end{array}$ & $\begin{array}{l}2.77 \div 2.95 \\
7.58 \div 7.70 \\
\end{array}$ & $\begin{array}{l}24 \\
16 \\
\end{array}$ & $\begin{array}{l}\text { F0-M5, pec } \\
\text { F5-M5, pec }\end{array}$ & $\begin{array}{l}\text { II-III, sd } \\
\text { III }\end{array}$ & $\begin{array}{l}\mathrm{CCD} \\
\mathrm{CCD} \\
\end{array}$ & $\begin{array}{l}\text { abs } \\
\text { abs }\end{array}$ \\
\hline 62 & O'Connell & 1973 & $3300-10800$ & & 20,30 & $14-760$ & $49 \mathrm{~ms}$ & O5-M8 & $\mathrm{I}-\mathrm{V}, \mathrm{sd}$ & Sp.Sc. & rel \\
\hline 64 & Prugniel \& Soubiran & 2001 & $\begin{array}{l}4100-6800 \\
4100-6800 \\
\end{array}$ & & $\begin{array}{l}42000 \\
10000 \\
\end{array}$ & $\begin{array}{l}0.05 \\
0.2 \\
\end{array}$ & $\begin{array}{l}708 \\
708 \\
\end{array}$ & $\begin{array}{l}\mathrm{O} 8-\mathrm{M} 2 \\
\mathrm{O} 8-\mathrm{M} 2 \\
\end{array}$ & $\begin{array}{l}\text { II-V } \\
\text { II-V }\end{array}$ & $\begin{array}{l}\mathrm{CCD} \\
\mathrm{CCD} \\
\end{array}$ & $\begin{array}{c}\text { norm } \\
\text { rel }\end{array}$ \\
\hline 65 & Santos et al. & 2001 & $\begin{array}{l}7090-10200 \\
3512-10226 \\
\end{array}$ & & $3-20$ & $\begin{array}{l}3 \\
3 \\
\end{array}$ & $\begin{array}{l}72 \\
22 \mathrm{~ms} \\
\end{array}$ & $\begin{array}{l}\text { B2-M5 } \\
\text { B8-M5 }\end{array}$ & $\begin{array}{l}\mathrm{I}-\mathrm{V} \\
\mathrm{I}-\mathrm{V}\end{array}$ & $\begin{array}{l}\text { several } \\
\text { several }\end{array}$ & $\begin{array}{l}\text { rel } \\
\text { rel }\end{array}$ \\
\hline 66 & Serote Roos et al. & 1996 & $\begin{array}{l}4800-8920 \\
5000-9780 \\
\end{array}$ & $\begin{array}{l}0.43 \\
3.3 \\
\end{array}$ & $\begin{array}{l}1.25 \\
8.5 \\
\end{array}$ & $\begin{array}{l}0.4 \\
3.3 \\
\end{array}$ & $\begin{array}{r}21 \\
7 \\
\end{array}$ & $\begin{array}{l}\text { B3-M5 } \\
\text { F2-M1 }\end{array}$ & $\begin{array}{l}\mathrm{I}-\mathrm{V} \\
\mathrm{III}, \mathrm{V}\end{array}$ & $\begin{array}{c}\text { Reticon } \\
\text { CCD }\end{array}$ & $\begin{array}{l}\text { rel } \\
\text { rel }\end{array}$ \\
\hline 67 & Silva \& Cornell & 1992 & $3510-8930$ & & & 5 & $72 \mathrm{~ms}$ & O5-M6 & $\mathrm{I}-\mathrm{V}$ & $\mathrm{CCD}$ & rel \\
\hline 68 & Soubiran et al. & 1998 & $4400-6800$ & & 42000 & 0.05 & 211 & A4-M2 & $\mathrm{II}-\mathrm{V}$ & $\mathrm{CCD}$ & counts \\
\hline 69 & Spinrad \& Taylor & 1969 & $\begin{array}{l}3880-7400 \\
7980-10700 \\
\end{array}$ & & $\begin{array}{l}16,32 \\
16,32 \\
\end{array}$ & $\begin{array}{c}27-1100 \\
\text { DW }\end{array}$ & $\begin{array}{r}229 \\
82 \\
\end{array}$ & F8-M3 & III-IV & $\begin{array}{l}\text { Sp.Sc. } \\
\text { Sp.Sc. }\end{array}$ & $\begin{array}{l}\text { rel } \\
\text { rel }\end{array}$ \\
\hline 70 & Stickland & 1971 & $3350 \cdots 7782$ & & 48,64 & $50-700$ & 29 & O9-K7 & $\mathrm{V}$ & Sp.Sc. & rel \\
\hline 71 & Stone & 1977 & $3200-8370$ & & 49,98 & $50-450$ & 16 & $\mathrm{O}-\mathrm{G} 2$, pec & III-V, sd & Sp.Sc. & abs \\
\hline 72 & Stone & 1996 & $4040-8800$ & 5.5 & & 5 & 107 & O-M5, WD & III-V, sd & $\mathrm{CCD}$ & abs \\
\hline 73 & Stritzinger et al. & 2005 & $3050-11000$ & $2.85,5.34$ & $8.6,16.4$ & 2.9 & 108 & & & $\mathrm{CCD}$ & abs \\
\hline 74 & Takeda et al. & 2005 & $5000 \cdots 8800$ & & 70000 & $0.02-0.04$ & 161 & $\mathrm{~F} 2-\mathrm{K} 2$ & III-V & $\mathrm{CCD}$ & norm \\
\hline 77 & Tereshchenko & 1996 & $3200-7600$ & & 50 & 50 & 27 & M0-M8 & I-III & Sp.Sc. & abs \\
\hline 78 & Tereshchenko & 1999 & $3200-7600$ & & 50 & 50 & 16 & $\mathrm{C}, \mathrm{S}$ & & Sp.Sc. & abs \\
\hline 79 & Tereshchenko & 2001 & $3125-7575$ & & 50 & 50 & 24 & B8.5-G5 & IV-V & Sp.Sc. & abs \\
\hline 80 & Tereshchenko & 2002 & $3125-7575$ & & 50 & 50 & 41 & B9.5-G6 & IV-V & Sp.Sc. & abs \\
\hline 81 & Tinney \& Reid & 1998 & $6400-9100$ & $0.19 \div 0.27$ & 18750 & 0.19 & 4 & O9.5, M8, M9 & $\mathrm{V}$ & $\mathrm{CCD}$ & rel \\
\hline 82 & Torres-Dodgen \& Weaver & 1993 & $5800-8900$ & $5.7 \div 6.8$ & 15.5 & $5.7 \div 6.8$ & 60 & $\mathrm{O} 4-\mathrm{M} 3$ & $\mathrm{I}, \mathrm{III}, \mathrm{V}$ & Reticon & abs \\
\hline 83 & Torres-Dodgen \& Massey & 1988 & $3400-7300$ & & $10-15$ & $2-3$ & 173 & WR & & Vidicon & abs \\
\hline 84 & Torres \& Massey & 1987 & $3100-7200$ & 125 & 9 & $1.3-2.8$ & 20 & WR & & Reticon & abs \\
\hline 85 & Tur et al. & 1995 & $3200-8000$ & & 50 & 100 & 7 & $\mathrm{Be}$ & & Sp.Sc. & rel \\
\hline 86 & Valdes et al. & 2004 & $3460-9464$ & 0.4 & 1.2 & 0.4 & 1273 & $\mathrm{~A} 0-\mathrm{M} 8, \mathrm{C}, \mathrm{S}$ & $\mathrm{I}-\mathrm{V}, \mathrm{sd}$ & $\mathrm{CCD}$ & rel \\
\hline 87 & Walborn \& Fitzpatrick & 1990 & $3800-4900$ & 0.5 & 1.5 & 0.4 & 78 & $\mathrm{O}, \mathrm{B}$ & $\mathrm{I}-\mathrm{V}$ & 2D-ph.c. & norm \\
\hline 88 & Walborn \& Fitzpatrick & 2000 & $3800-4900$ & 0.5 & 1.5 & 0.4 & 21 & $\mathrm{O}, \mathrm{B}, \mathrm{WR}$ & & 2D-ph.c. & norm \\
\hline 89 & Wallace \& Hinkle & 1996 & $8690-9300$ & & 56000 & $0.06 \div 0.07$ & 1 & $\alpha$ Boo $(\mathrm{K} 1)$ & III & FTS & norm \\
\hline 90 & Weaver et al. & 1995 & $5750-8950$ & 6.8 & 15.5 & $5.7 \div 6.9$ & 43 & $\mathrm{~A}$ & $\mathrm{I}-\mathrm{V}$ & Reticon & abs \\
\hline 91 & Worthey \& Ottaviani & 1997 & $3900-6600$ & & $8 \div 10$ & 1.25 & 460 & B0.5-M8 & $\mathrm{I}-\mathrm{V}, \mathrm{sd}$ & IDS & \\
\hline 92 & Zwitter \& Munari & 1994 & $4600-9000$ & 7.8 & 18 & $7.5 \div 7.8$ & 41 & $\mathrm{CV}$ & & $\mathrm{CCD}$ & abs \\
\hline 93 & Zwitter \& Munari & 1995 & $3300-9150$ & & 7 & $2.85 \div 3.0$ & 25 & $\mathrm{CV}$ & & $\mathrm{CCD}$ & abs \\
\hline 94 & Zwitter \& Munari & 1996 & $3270-9000$ & & 7 & $2.76 \div 2.95$ & 38 & $\mathrm{CV}$ & & $\mathrm{CCD}$ & abs \\
\hline
\end{tabular}

Ultraviolet libraries with data in electronic form

\begin{tabular}{|c|c|c|c|c|c|c|c|c|c|c|}
\hline 95 & Ayres & 2003 & $1150-3000$ & 30000,110000 & 0.012 & 52 & F0-M5.5, T Tau & $\mathrm{I}-\mathrm{V}, \mathrm{sd}$ & STIS & abs \\
\hline 96 & Brandt et al. & 1995 & 1979-3300 & $20000-35000$ & $0.021 \div 0.025$ & 1 & $\alpha$ Ori (M2) & Iab & GHRS & abs \\
\hline 97 & Brandt et al. & 1998 & $1180-1777$ & $17000-21000$ & $0.017 \div 0.018$ & 1 & $10 \mathrm{Lac}(\mathrm{O} 9)$ & $\mathrm{V}$ & GHRS & $\mathrm{abs}$ \\
\hline 98 & Brandt et al. & 1999 & $1249 \cdots 2688$ & $75000-93000$ & $0.003 \div 0.007$ & 1 & $\chi$ Lupi (B9.5p+A & & GHRS & abs \\
\hline
\end{tabular}


R. Sordo and U. Munari: The Asiago Database of Spectroscopic Databases (ADSD), Online Material p 4

Table 2. (continued)

\begin{tabular}{|c|c|c|c|c|c|c|c|c|c|c|c|}
\hline $\begin{array}{c}\text { Fig } \\
\text { N. }\end{array}$ & $\begin{array}{r}\text { Authors an } \\
\text { of publica }\end{array}$ & & $\begin{array}{c}\text { Spectral } \\
\text { Range } \\
(\AA) \\
\end{array}$ & $\begin{array}{c}\mathbf{D} \\
(\mathbf{\AA} / \mathbf{p} \mathbf{i x}) \\
(\mathbf{\AA} / m m) \\
\end{array}$ & $\begin{array}{c}\mathbf{R} \text { or } R_{P} \\
\text { (A) }\end{array}$ & $\begin{array}{c}\text { Samplig } \\
\text { (A) }\end{array}$ & $\begin{array}{c}\mathbf{N} \\
\text { Stars }\end{array}$ & Spectrum & $\begin{array}{l}\text { Lum. } \\
\text { Class }\end{array}$ & $\begin{array}{c}\text { detector/ } \\
\text { instr. }\end{array}$ & data \\
\hline 99 & Cacciari & 1985 & $\begin{array}{l}1900-3200 \\
1200-1900 \\
\end{array}$ & & & $\begin{array}{l}2.66 \\
1.67 \\
\end{array}$ & $\begin{array}{l}36 \\
19 \\
\end{array}$ & $\begin{array}{l}\text { popII } \\
\text { popII }\end{array}$ & $\begin{array}{l}\text { III-V } \\
\text { III-V }\end{array}$ & $\begin{array}{l}\text { IUE } \\
\text { IUE }\end{array}$ & $\begin{array}{l}\text { abs } \\
\text { abs }\end{array}$ \\
\hline 100 & Code \& Meade & 1979 & $1160-3600$ & & 12,22 & 20 & 164 & O5-M2, WR & $\mathrm{I}-\mathrm{V}$ & Sp.Sc. & abs \\
\hline 101 & Craig et al. & 1997 & $70-760$ & & $0.5-2$ & $0.05-2$ & 96 & $\mathrm{~B} 2-\mathrm{M} 5, \mathrm{WD}, \mathrm{CV}$ & $\mathrm{I}-\mathrm{V}$ & EUVE & abs \\
\hline 102 & Fanelli et al. & 1992 & $1205-3185$ & & 6 & $1-2$ & $56 \mathrm{~ms}$ & O3-M4 & $\mathrm{I}-\mathrm{V}$ & IUE & rel \\
\hline 103 & Heck et al. & 1984 & $1150-3200$ & & 7 & 2 & 229 & O3-G5 & $\mathrm{I}-\mathrm{V}$ & IUE & abs \\
\hline 104 & Henize et al. & 1979 & $1300-4200$ & & & $2-20$ & 494 & & & plate & abs \\
\hline 105 & Holberg et al. & 1998 & $1150-1950$ & & 10000 & 0.03 & 55 & WD & & IUE & abs \\
\hline 106 & Holberg et al. & 2003 & $1150-3200$ & & 6 & $1.67,2.66$ & 336 & WD & & IUE & abs \\
\hline 107 & Jamar et al. & 1976 & $1360-2540$ & 36 & & 20 & 1356 & O4-K0, WR & $\mathrm{I}-\mathrm{V}$ & Sp.Sc. & abs \\
\hline 108 & Macau-Hercot et al. & 1978 & $1360-2540$ & 36 & & 10 & 435 & $\mathrm{O}-\mathrm{M}$ & $\mathrm{I}-\mathrm{V}$ & Sp.Sc. & abs \\
\hline 109 & Meier et al. & 1994 & $1150-1980$ & & 6 & 1.68 & 32 & Symb. stars & & IUE & $\mathrm{abs}$ \\
\hline 110 & Pellerin et al. & 2002 & $912-1185$ & & 20000 & $0.006,0.007$ & 45 & $\mathrm{O} 2-\mathrm{B} 3$ & $\mathrm{I}-\mathrm{V}$ & FUSE & norm \\
\hline 111 & Robert et al. & 1993 & $1205-1850$ & & & 0.75 & $51 \mathrm{~ms}$ & $\mathrm{O}, \mathrm{WR}$ & $\mathrm{I}-\mathrm{V}$ & IUE & norm \\
\hline 112 & Rogerson & 1985 & $970-1501$ & & $0.05,0.1$ & $0.020-0.053$ & 1 & $\gamma$ Peg (B2) & IV & Sp.Sc. & counts \\
\hline 113 & Rogerson & 1987 & $1649-3170$ & & 0.1 & $0.038-0.054$ & 1 & $\alpha \mathrm{CMa}(\mathrm{A} 1)$ & $\mathrm{V}$ & Sp.Sc. & counts \\
\hline 114 & Rogerson & 1989 & $2000-3187$ & & 0.1 & $0.038-0.048$ & 1 & $\alpha \operatorname{Lyr}(\mathrm{A} 0)$ & $\mathrm{V}$ & Sp.Sc. & counts \\
\hline 115 & Rogerson \& Upson & 1977 & $949-1560$ & & $0.05,0.1$ & $0.020-0.053$ & 1 & $\tau$ Sco (B0) & $\mathrm{V}$ & Sp.Sc. & counts \\
\hline 116 & Rogerson \& Upson & 1982 & $949-1561$ & & $0.05,0.1$ & $0.020-0.053$ & 1 & $\beta$ Ori (B8) & $\mathrm{Ia}$ & Sp.Sc. & counts \\
\hline 117 & Snow \& Jenkins & 1977 & $1000-1450$ & & 0.2 & 0.2 & 60 & $\mathrm{O} 4-\mathrm{A} 1$ & $\mathrm{I}-\mathrm{V}$ & Sp.Sc. & abs \\
\hline 118 & Upson \& Rogerson & 1980 & $999-1467$ & & $0.05,0.1$ & $0.020-0.053$ & 1 & 1 Her (B3) & IV & Sp.Sc. & counts \\
\hline 119 & Valenti et al. & 2000 & $1150-1980$ & & 6 & 1.67 & 142 & $\mathrm{~T} \mathrm{Tau}, \mathrm{Ae} / \mathrm{Be}$ & & IUE & abs \\
\hline 120 & Valenti et al. & 2003 & $1850-3200$ & & 6 & 2.66 & 191 & T Tau, Ae/Be & & IUE & abs \\
\hline 121 & Walborn et al. & 1985 & $1150-1950$ & & 0.25 & 0.25 & 98 & O3-B1, WR & $\mathrm{I}-\mathrm{V}, \mathrm{sd}$ & IUE & norm \\
\hline 122 & Walborn et al. & 1995 & $1150-1950$ & & 0.25 & 0.25 & 86 & O9-B8 & $\mathrm{I}-\mathrm{V}$ & IUE & norm \\
\hline 123 & Walborn et al. & 2002 & $905-1187$ & & 20000 & 0.25 & 47 & $\mathrm{O} 2-\mathrm{B} 1.5$ & $\mathrm{I}-\mathrm{V}$ & FUSE & abs \\
\hline 124 & Willis et al. & 2004 & $905-1187$ & & 20000 & $0.094 \div 0.109$ & 21 & WN, WC & & FUSE & abs \\
\hline 125 & Wood et al. & 1996 & $1205 \cdots 2806$ & & $17000 \div 21000$ & $0.006-0.02$ & 1 & $\alpha$ CMi (F5) & IV-V & & abs \\
\hline 126 & Wu et al. & 1996 & $\begin{array}{l}1150-3200 \\
1150-3200 \\
\end{array}$ & & $\begin{array}{l}6 \\
6 \\
\end{array}$ & $\begin{array}{l}1.67,2.66 \\
1.67,2.66 \\
\end{array}$ & $\begin{array}{r}476 \\
38 \\
\end{array}$ & $\begin{array}{l}\text { O3-M7 } \\
\text { O-B, WD }\end{array}$ & $\begin{array}{l}\mathrm{I}-\mathrm{V} \\
\text { sd }\end{array}$ & IUE & $\begin{array}{l}\text { abs } \\
\text { abs }\end{array}$ \\
\hline
\end{tabular}

Infrared libraries with data in electronic form

\begin{tabular}{|c|c|c|c|c|c|c|c|c|c|c|}
\hline 127 & Arnaud et al. & 1989 & $2.0-2.45$ & & 200 & 80 & 73 & F3-M8 & II-V & rel \\
\hline \multirow[t]{2}{*}{128} & Burgasser et al. & 2002 & $1.0-2.5$ & 49,59 & $60-120$ & 49,59 & 16 & $\mathrm{~T}$ & $\mathrm{~V}$ & abs \\
\hline & & & $1.2-2.35$ & $4.4 \div 8.8$ & 1200 & $4.4 \div 8.8$ & 14 & $\mathrm{~T}$ & $\mathrm{~V}$ & abs \\
\hline 129 & Burgasser et al. & $2003 \mathrm{~b}$ & $1.2-2.35$ & $4.4 \div 8.8$ & 1200 & $4.4 \div 8.8$ & 6 & $\mathrm{~T} 0-\mathrm{T} 8$ & $\mathrm{~V}$ & abs \\
\hline 130 & Cohen et al. & 1995 & $1.2-35$ & & 30 & $200-1000$ & 5 & $\mathrm{~K} 0-\mathrm{M} 2$ & III & abs \\
\hline 131 & Cohen et al. & $1996 \mathrm{a}$ & $3-35$ & & 30 & $200-3100$ & 3 & $\mathrm{~K} 2-\mathrm{G} 2$ & III,V & abs \\
\hline 132 & Cohen et al. & $1996 \mathrm{~b}$ & $1.2-35$ & & & $30-3100$ & 5 & $\mathrm{~K} 1-\mathrm{M} 3$ & III & abs \\
\hline 133 & Dallier et al. & 1996 & $1.57-1.64$ & & $1500-2000$ & 3 & 37 & $\mathrm{O} 7-\mathrm{M} 2$ & $\mathrm{I}, \mathrm{III}, \mathrm{V}$ & rel \\
\hline \multirow[t]{2}{*}{134} & Förster Schreiber & 2000 & $2.18-2.45$ & & 2000 & 5 & 17 & $\mathrm{~K} 0-\mathrm{M} 4.5, \mathrm{C}$ & I-III & rel \\
\hline & & & $1.9-2.4$ & & 830 & 10 & 16 & G8.5-M4 & I-III & rel \\
\hline 135 & Hanson et al. & 1996 & $2.0-2.2$ & & $800-3000$ & 8 & 180 & O3-M3, pec & $\mathrm{I}-\mathrm{V}$ & norm \\
\hline 136 & Hinkle et al. & 1995 & $0.9-5.3$ & & 100000 & $0.03 \div 0.3$ & 1 & $\alpha$ Boo (K1) & III & norm \\
\hline 137 & IRAS & 1986 & $7.7-22.6$ & & $20 \div 60$ & $1000-2000$ & 5425 & & & abs \\
\hline 138 & Johnson \& Mendez & 1970 & $1-4$ & & $8 \mathrm{~cm}^{-1}$ & $60 \div 100$ & 30 & A0-M8, pec, C & $\mathrm{I}-\mathrm{V}$ & counts \\
\hline 139 & Kleinmann \& Hall & 1986 & $2.02-2.4$ & & $1.6 \mathrm{~cm}^{-1}$ & 0.9 & 26 & F8-M7 & $\mathrm{I}, \mathrm{III}, \mathrm{V}$ & rel \\
\hline 140 & Lançon et al. & 1992 & $1.43-2.5$ & & 500 & $4-12$ & 56 & O6-M8 & $\mathrm{I}-\mathrm{V}$ & rel \\
\hline 141 & Lucas et al. & 2001 & $1.43-2.5$ & & $330-440$ & $25 \div 50$ & 23 & brown dwarfs & & counts \\
\hline 142 & Malkan et al. & 2002 & $1.08-1.35$ & & 400 & 11.5 & 105 & O9.5-M7 & $\mathrm{I}-\mathrm{V}$ & rel \\
\hline \multirow[t]{2}{*}{143} & McLean et al. & 2003 & $0.94-2.31$ & & 2000 & $2-4$ & 16 & M6-T8 & & abs \\
\hline & & & $1.13-2.31$ & & 2000 & $2-4$ & 37 & M6-T8 & & abs \\
\hline 144 & Meyer et al. & 1998 & $1.51-1.78$ & & 3000 & 3 & 85 & O7-M5 & $\mathrm{I}-\mathrm{V}$ & norm \\
\hline 145 & Testi et al. & 2001 & $0.85-2.45$ & & 100 & $30 \div 110$ & 26 & $\mathrm{~L}$ & $\mathrm{~V}$ & rel \\
\hline
\end{tabular}


R. Sordo and U. Munari: The Asiago Database of Spectroscopic Databases (ADSD), Online Material p 5

Table 2. (continued)

\begin{tabular}{|c|c|c|c|c|c|c|c|c|c|c|}
\hline $\begin{array}{l}\text { Fig } \\
\text { N. }\end{array}$ & $\begin{array}{r}\text { Authors a } \\
\text { of publi }\end{array}$ & & $\begin{array}{c}\text { Spectral } \\
\text { Range } \\
(\AA) \\
\end{array}$ & 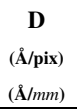 & $\begin{array}{c}\mathbf{R} \text { or } R_{P} \\
\quad(\AA)\end{array}$ & $\begin{array}{c}\text { Samplig } \\
(\AA)\end{array}$ & $\begin{array}{c}\text { N. } \\
\text { Stars }\end{array}$ & Spectrum & $\begin{array}{l}\text { Lum. } \\
\text { Class }\end{array}$ & $\begin{array}{l}\text { detector/ data } \\
\text { instr. }\end{array}$ \\
\hline 146 & Wallace et al. & 2000 & $1.05-1.34$ & & 3000 & $1 \div 2$ & 88 & O7-M6 & $\mathrm{I}-\mathrm{V}$ & rel \\
\hline 147 & Wallace \& Hinkle & 1997 & $2-2.4$ & & 3000 & $2 \div 3$ & 119 & O4-M8, pec & $\mathrm{I}-\mathrm{V}$ & rel \\
\hline
\end{tabular}

Combined libraries with data in electronic form

\begin{tabular}{|c|c|c|c|c|c|c|c|c|c|c|}
\hline 148 & Bohlin et al. & 2001 & $1140-10250$ & & & $0.5-4.9$ & 32 & $\mathrm{O}, \mathrm{B}, \mathrm{A}, \mathrm{WD}$ & & abs \\
\hline 149 & Pickles & 1998 & $1150-25000$ & & 500 & 5 & $131 \mathrm{~ms}$ & O5-M10 & $\mathrm{I}-\mathrm{V}$ & rel \\
\hline 150 & Sviderskiene & 1988 & $1200-10500$ & & & 50 & $98 \mathrm{~ms}$ & $\mathrm{O}-\mathrm{M}$ & $\mathrm{I}-\mathrm{V}$ & rel \\
\hline 151 & Knapp et al. & 2004 & $8400-25000$ & & $20-50$ & 6,13 & 55 & $\mathrm{~L}, \mathrm{~T}$ & & abs \\
\hline 152 & Lançon \& Wood & 2000 & $\begin{array}{r}10000-25000 \\
5000-10000 \\
\end{array}$ & 9.7 & $\begin{array}{c}1100 \\
30 \div 40 \\
\end{array}$ & $\begin{array}{l}5 \\
5 \\
\end{array}$ & $\begin{array}{l}57 \\
71 \\
\end{array}$ & $\begin{array}{l}\text { K0-M9, C } \\
\text { K0-M9, C }\end{array}$ & & $\begin{array}{l}\text { counts } \\
\text { counts }\end{array}$ \\
\hline 153 & Lançon \& Mouhcine & 2002 & $5100-24900$ & & & 5 & $17 \mathrm{~ms}$ & AGB & & rel \\
\hline
\end{tabular}


R. Sordo and U. Munari: The Asiago Database of Spectroscopic Databases (ADSD), Online Material p 6

Table 3. The list of optical, ultraviolet and infrared libraries that give access to the spectra in printed tabular form and that are included in ADSD. The first column lists the number of the figure on the ADSD web site that provide access to the card of the given library and that is included in the printable book-size full version of ADSD.

\begin{tabular}{c|c|c|c|c|c|c|cc|c}
\hline $\begin{array}{c}\text { Fig } \\
\text { N. }\end{array}$ & $\begin{array}{c}\text { Authors and year } \\
\text { of publication }\end{array}$ & $\begin{array}{c}\text { Spectral } \\
\text { Range } \\
(\AA)\end{array}$ & $\begin{array}{c}\text { D } \\
(\AA / \text { / pix }) \\
(\AA / m m)\end{array}$ & $\begin{array}{c}\text { R or } R_{P} \\
(\AA)\end{array}$ & $\begin{array}{c}\text { Samplig } \\
(\AA)\end{array}$ & $\begin{array}{c}\text { N. } \\
\text { Stars }\end{array}$ & $\begin{array}{c}\text { Spectrum } \\
\text { Lum. } \\
\text { Class }\end{array}$ & $\begin{array}{c}\text { detector/ } \\
\text { instr. }\end{array}$ \\
\hline \hline
\end{tabular}

\section{Optical libraries with data in printed form}

\begin{tabular}{|c|c|c|c|c|c|c|c|c|c|c|c|}
\hline 154 & Bahner & 1963 & $3200-6400$ & & 54 & $100-430$ & 25 & O9-F5 & $\mathrm{I}-\mathrm{V}$ & Sp.Sc. & rel \\
\hline 155 & Baldwin \& Stone & 1984 & $6056-10400$ & & 80 & $90-1125$ & 18 & A-G, WD & & several & abs \\
\hline 156 & Beavers \& Cook & 1980 & $3500-4400$ & & 10 & DW & 116 & O9-G1 & IV-V & Sp.Sc. & rel \\
\hline 157 & Böhm-Vitense \& Johnson & 1977 & $3500-8080$ & & 40 & $60-400$ & 43 & A4-F5 & IV-V & Sp.Sc. & abs \\
\hline 158 & Christensen & 1978 & $3450-10800$ & & 20,30 & DW & 65 & & & Sp.Sc. & $\mathrm{abs}$ \\
\hline 159 & Cochran & 1981 & $4650-10000$ & & 42 & 20 & 16 & B0-A3 & $\mathrm{I}-\mathrm{V}$ & Reticon & abs \\
\hline 160 & Danziger \& Dickens & 1967 & $3400-10800$ & & 50 & $60-1200$ & 9 & A2-F4, pec & II-V & Sp.Sc. & $\mathrm{abs}$ \\
\hline 161 & Davis \& Webb & 1974 & $3300-8080$ & & 50 & $60-430$ & 33 & O5-F8 & $\mathrm{I}-\mathrm{V}$ & Sp.Sc. & rel \\
\hline 162 & Dickens \& Penny & 1971 & $3500-8000$ & & 40,80 & $60-530$ & 31 & A3-F7 & II-V & Sp.Sc. & abs \\
\hline 163 & Fawley & 1977 & $4000-11000$ & & 48,32 & DW & 16 & G8-M9 & $\mathrm{I}$ & several & abs \\
\hline 164 & Faÿ et al. & 1974 & $3300-7000$ & & 30 & $50-400$ & 25 & G0-M5 & $\mathrm{I}, \mathrm{III}, \mathrm{V}$ & Sp.Sc. & rel \\
\hline 165 & Goraya & 1984 & $3500-7500$ & & 50 & 100 & 7 & B1e-B8e & III-V & Sp.Sc. & rel \\
\hline 166 & Goraya & 1986 & $3200-8000$ & & 50 & 100 & 26 & B0e-B9e & III-V & Sp.Sc. & rel \\
\hline 167 & Goraya \& Gurm & 1987 & $3200-8000$ & & 50 & 100 & 8 & B2e-B4e & III-V & Sp.Sc. & rel \\
\hline 168 & Goraya \& Tur & 1988 & $3200-8000$ & & 50 & 100 & 9 & B1e-B3e & III-V & Sp.Sc. & rel \\
\hline 169 & Gutiérrez-Moreno et al. & 1988 & $3200-8370$ & & 40,80 & $50-400$ & 15 & B0-G8 & IV-V & Sp.Sc. & $\mathrm{abs}$ \\
\hline 170 & Hamuy et al. & 1992 & $3350-7550$ & & $40-98$ & DW & 19 & B-G, WD, C & & $\mathrm{CCD}$ & $\mathrm{abs}$ \\
\hline 171 & Hamuy et al. & 1994 & $6050-10400$ & & 80,98 & DW & 20 & B-F, WD, pec & & $\mathrm{CCD}$ & abs \\
\hline 172 & Hayes & 1970 & $3200-10870$ & & 30,45 & $50-1125$ & 12 & B0-A1 & I, III-V & Sp.Sc. & rel \\
\hline 173 & Hayes \& Philip & 1983 & $3410-6840$ & & 40 & $160-360$ & 20 & A & & Sp.Sc. & rel \\
\hline 174 & Hickson \& Mulrooney & 1998 & $3500-9200$ & & 15 & 50 & 21 & & & $\mathrm{CCD}$ & $\mathrm{abs}$ \\
\hline 175 & Hua et al. & 1983 & $3200-8100$ & $2.5,5$ & & $50-450$ & 6 & WR & & Sp.Sc. & abs \\
\hline 176 & Jeffers \& Weller & 1985 & $3800-7000$ & & 7,15 & 10 & 10 & WN, WC & & Vidicon & rel \\
\hline 177 & Kaiser & 1987 & $3200-8500$ & & 50 & 50 & 26 & $\mathrm{Be}$ & III-V & Sp.Sc. & abs \\
\hline 178 & Kharitonov et al. & 1997 & $3125-7175$ & & & 50 & 60 & O6-A3, pec & $\mathrm{I}-\mathrm{V}$ & Sp.Sc. & $\mathrm{abs}$ \\
\hline 179 & Knyazeva \& Kharitonov & 2000 & $3225-7575$ & & & 50 & $16 \mathrm{~ms}$ & O9.5-G2 & I-IV & Sp.Sc. & rel \\
\hline 180 & Kuhi & 1966 & $3200-11000$ & & 40,50 & $35-840$ & 21 & WN, WC & & Sp.Sc. & rel \\
\hline 181 & Kuhi & 1974 & $3320-7500$ & & 48,64 & & 25 & T Tau var. & & Sp.Sc. & abs \\
\hline 182 & Lane \& Lester & 1980 & $3400-8090$ & & 40,80 & $50-450$ & 13 & $\mathrm{Am}$ & & Sp.Sc. & rel \\
\hline 183 & Lockwood & 1973 & 9711-10834 & & 32 & DW & 21 & M2-M10 & & Sp.Sc. & abs \\
\hline 184 & Massey \& Gronwall & 1990 & $7350 \div 10200$ & & 50 & 50 & 11 & O-A, DA & $\mathrm{V}, \mathrm{sd}$ & $\mathrm{CCD}$ & abs \\
\hline 185 & Oke & 1964 & $3390-10800$ & & 50 & $60-900$ & 13 & O9.5-A1 & $\mathrm{I}-\mathrm{V}$ & Sp.Sc. & abs \\
\hline 186 & Oke & 1990 & $3200-9200$ & & 40 & $5-390$ & 25 & $\mathrm{O}-\mathrm{G}, \mathrm{WD}$ & IV-V, sd & $\mathrm{CCD}$ & abs \\
\hline 187 & Oke, Gunn & 1983 & $3080-12000$ & & 40 & $20-860$ & 4 & $\mathrm{~F}$ & $\mathrm{sd}$ & Sp.Sc. & $\mathrm{abs}$ \\
\hline 188 & Panek & 1977 & $3600-4200$ & & 10 & 5 & 7 & $\mathrm{~A} 0-\mathrm{A} 5$ & III-V & Sp.Sc. & abs \\
\hline 189 & Philip \& Hayes & 1983 & $3450-6790$ & & 40,80 & $50-380$ & 25 & B5-F6 & & Sp.Sc. & rel \\
\hline 190 & Schild et al. & 1971 & $3300-6050$ & & 50,100 & $60-330$ & 54 & O9.5-A7 & $\mathrm{I}-\mathrm{V}$ & Sp.Sc. & abs \\
\hline 191 & Schild & 1978 & $3200-8572$ & & 50 & $50-450$ & 61 & $\mathrm{Be}$ & & Sp.Sc. & $\mathrm{abs}$ \\
\hline 192 & Schild \& Chaffee & 1971 & $3200-5556$ & & 50 & $50-295$ & 19 & B & III-V & Sp.Sc. & rel \\
\hline 193 & Schmidt-Kaler \& Oestreicher & 1999 & $3260-7770$ & & 10 & 10 & 6 & $\mathrm{~B} 2-\mathrm{A} 0$ & & Rubikon & abs \\
\hline 194 & Spinrad \& Taylor & 1971 & $\begin{array}{l}3300-10700 \\
3300-10700 \\
\end{array}$ & & $\begin{array}{l}16,32 \\
16,32 \\
\end{array}$ & $\begin{array}{l}\text { DW } \\
\text { DW }\end{array}$ & $\begin{array}{c}120 \\
34 \mathrm{~ms}\end{array}$ & $\begin{array}{l}\text { O9-M9, C, WD } \\
\text { O-M, C, WD }\end{array}$ & $\begin{array}{l}\text { III-V } \\
\text { III-V }\end{array}$ & $\begin{array}{l}\text { Sp.Sc. } \\
\text { Sp.Sc. }\end{array}$ & $\begin{array}{l}\text { rel } \\
\text { rel }\end{array}$ \\
\hline 195 & Stone \& Baldwin & 1983 & $3200-8280$ & & 40,80 & $50-310$ & 18 & A-G, WD & & several & abs \\
\hline 196 & Taylor & 1970 & $4037-8800$ & & 16,32 & DW & 280 & G2-K7 & $\mathrm{V}$ & Sp.Sc. & rel \\
\hline 197 & Taylor et al. & 1972 & $3880-7400$ & & 16,32 & DW & 34 & G5-M0 & II-V & Sp.Sc. & rel \\
\hline 198 & Taylor & 1979 & $5840-11000$ & & $32-96$ & $150-1120$ & 24 & & & Sp.Sc. & abs \\
\hline 199 & Taylor & 1982 & $\begin{array}{l}4040-6180 \\
6110-10700\end{array}$ & & $\begin{array}{c}9.6-32 \\
32 \\
\end{array}$ & $\begin{array}{l}\text { DW } \\
\text { DW }\end{array}$ & $\begin{array}{l}14 \\
32 \\
\end{array}$ & & & $\begin{array}{l}\text { Sp.Sc. } \\
\text { Sp.Sc. }\end{array}$ & $\begin{array}{l}\text { abs } \\
\text { abs }\end{array}$ \\
\hline 200 & Taylor & $1984 \mathrm{~b}$ & $3300-10800$ & & 50 & $60-1120$ & 22 & B-A & & Sp.Sc. & abs \\
\hline 201 & Tüg & 1980 & $3200-8800$ & & $1.5-20$ & $50-350$ & 14 & O5-F0 & $\mathrm{I}-\mathrm{V}$ & Sp.Sc. & abs \\
\hline
\end{tabular}


R. Sordo and U. Munari: The Asiago Database of Spectroscopic Databases (ADSD), Online Material p 7

Table 3. (continued)

\begin{tabular}{c|c|c|c|c|c|c|cc|c}
\hline Fig & $\begin{array}{c}\text { Authors and year } \\
\text { of publication }\end{array}$ & $\begin{array}{c}\text { Spectral } \\
\text { Range } \\
(\AA)\end{array}$ & $\begin{array}{c}\mathbf{D} \\
(\AA / \mathrm{pix}) \\
(\AA / \mathrm{\Omega} / \mathrm{m})\end{array}$ & $\begin{array}{c}\mathbf{R} \text { or } R_{P} \\
(\AA)\end{array}$ & $\begin{array}{c}\text { Samplig } \\
(\AA)\end{array}$ & $\begin{array}{c}\mathbf{N} . \\
\text { Stars }\end{array}$ & $\begin{array}{c}\text { Spectrum } \\
\text { Cum. }\end{array}$ & $\begin{array}{c}\text { detector/ data } \\
\text { instr. }\end{array}$ \\
\hline \hline
\end{tabular}

Ultraviolet libraries with data in printed form

\begin{tabular}{|c|c|c|c|c|c|c|c|c|c|c|}
\hline 202 & Bohlin & 1986 & $1155-3195$ & & 5 & 5 & O-B3, pec & $\mathrm{IV}, \mathrm{V}$ & IUE & $\mathrm{abs}$ \\
\hline 203 & Brune et al. & 1979 & $912-3100$ & 15 & 10 & 5 & O4-B3, WR & $\mathrm{I}-\mathrm{V}$ & Sp.Sc. & abs \\
\hline 204 & Fanelli et al. & 1987 & $1230-1930$ & 20 & $10-100$ & $15 \mathrm{~ms}$ & O3-A7 & $\mathrm{I}, \mathrm{III}, \mathrm{V}$ & IUE & rel \\
\hline 205 & Lamers et al. & 1981 & $2060 \cdots 2880$ & $1.7-2.3$ & 0.4 & 220 & O4-G2, pec & $\mathrm{I}-\mathrm{V}$ & Sp.Sc. & $\mathrm{abs}$ \\
\hline 206 & Meade \& Code & 1980 & $\begin{array}{l}1160-1850 \\
1800-3600\end{array}$ & $\begin{array}{l}12 \\
22\end{array}$ & $\begin{array}{l}10 \\
20\end{array}$ & $\begin{array}{r}132 \\
34\end{array}$ & $\begin{array}{l}\mathrm{O} 7-\mathrm{A} 3 \\
\mathrm{O} 5-\mathrm{K} 0\end{array}$ & $\begin{array}{l}\mathrm{I}-\mathrm{V} \\
\mathrm{I}-\mathrm{V}\end{array}$ & $\begin{array}{l}\text { Sp.Sc. } \\
\text { Sp.Sc. }\end{array}$ & $\begin{array}{l}\text { abs } \\
\text { abs }\end{array}$ \\
\hline 207 & Papaj et al. & 1990 & $1380-2540$ & & 20 & $12 \mathrm{~ms}$ & O9.5-B5 & III, V & Sp.Sc. & rel \\
\hline 208 & Parsons \& Ake & 1993 & $\begin{array}{l}1300-3000 \\
2500-3000\end{array}$ & $\begin{array}{l}100 \\
100\end{array}$ & $\begin{array}{l}100 \\
100\end{array}$ & $\begin{array}{l}13 \mathrm{~ms} \\
35 \mathrm{~ms}\end{array}$ & $\begin{array}{l}\text { B0-F0 } \\
\text { G5-M6 }\end{array}$ & $\begin{array}{l}\mathrm{V} \\
\mathrm{I}-\mathrm{III}\end{array}$ & $\begin{array}{l}\text { IUE } \\
\text { IUE }\end{array}$ & $\begin{array}{l}\text { abs } \\
\text { abs }\end{array}$ \\
\hline 209 & Smith \& Willis & 1983 & $1150-3080$ & $6-9$ & 5,10 & 9 & WN, WC & & IUE & abs \\
\hline 210 & Wegner \& Swanson & 1991 & $1160-3200$ & 7 & 20 & 182 & WD & & IUE & $\mathrm{abs}$ \\
\hline 211 & Willis \& Wilson & 1978 & $1350-2550$ & 35 & 10 & 9 & WN, WC & & Sp.Sc. & $\mathrm{abs}$ \\
\hline 212 & Woods et al. & 1985 & $912-1600$ & 10 & 10 & 6 & O4-A1, WR & $\mathrm{I}, \mathrm{IIII}, \mathrm{V}$ & Sp.Sc. & $\mathrm{abs}$ \\
\hline
\end{tabular}

Infrared libraries with data in printed form

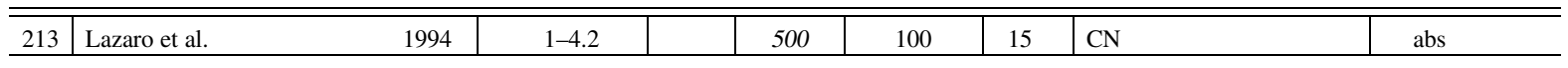


R. Sordo and U. Munari: The Asiago Database of Spectroscopic Databases (ADSD), Online Material p 8

Table 4. The list of optical, ultraviolet and infrared libraries that present the spectra in graphical form and that are included in ADSD.

\begin{tabular}{c|c|c|c|c|cc|c|}
\hline $\begin{array}{c}\text { Authors \& year } \\
\text { of publication }\end{array}$ & $\begin{array}{c}\text { Spectral } \\
\text { Range } \\
(\AA)\end{array}$ & $\begin{array}{c}\mathbf{D} \\
(\AA / \mathrm{pix}) \\
(\AA / \mathrm{mm})\end{array}$ & $\begin{array}{c}\mathbf{R} \text { o } \mathbf{R}_{P} \\
(\AA)\end{array}$ & $\begin{array}{c}\text { N. } \\
\text { Stars }\end{array}$ & $\begin{array}{c}\text { Spectrum } \\
\text { Lum. }\end{array}$ & $\begin{array}{c}\text { detector } / \\
\text { instr. }\end{array}$ & $\begin{array}{c}\text { data } \\
\text { Class }\end{array}$ \\
\hline \hline
\end{tabular}

Optical libraries with data in graphical form

\begin{tabular}{|c|c|c|c|c|c|c|c|c|c|}
\hline Allen & 1984 & $3400-7500$ & & $3-10$ & 100 & \multicolumn{2}{|l|}{ Symb. stars } & IPCS,IDS & norm \\
\hline Andrillat et al. & 1988 & $7500-8800$ & 50 & & 51 & \multicolumn{2}{|l|}{$\mathrm{Be}$} & Reticon & counts \\
\hline Andrillat et al. & 1994 & $3800 \cdots 10950$ & $33-260$ & $1.2-13.3$ & 1 & \multicolumn{2}{|l|}{ PU Vul (Symb. nova) } & $\mathrm{CCD}$ & rel \\
\hline Bessell & 1991 & $5000-10200$ & & 5,15 & 58 & M4-M10 & $\mathrm{V}$ & IDS, CCD & rel \\
\hline Brewer et al. & 1996 & $5500-6800$ & 1.55 & 3.1 & 87 & $\mathrm{C}, \mathrm{S}, \mathrm{M}$ & III & CCD & rel \\
\hline Bruch & 1989 & $3900-7400$ & $3.25-13$ & 24,31 & 12 & $\mathrm{CV}$ & $\mathrm{V}$ & CCD,Reticon & rel \\
\hline Bruch, Schimpke & 1992 & $3800-7200$ & 3.5 & $7.6 \div 14.4$ & 25 & $\mathrm{CV}$ & $\mathrm{V}$ & $\mathrm{CCD}$ & abs \\
\hline Chan & 1993 & $4670 \cdots 6350$ & 15,30 & & 11 & C & & $\mathrm{CCD}$ & counts \\
\hline Chen et al. & 2001 & $3800-5000$ & 100 & 4 & 12 & $\mathrm{CV}$ & & Reticon & norm \\
\hline Dawson et al. & 2000 & $4500-10000$ & & 900 & 10 & K-M5.2 & $\mathrm{V}, \mathrm{sd}$ & $\mathrm{CCD}$ & rel \\
\hline Dopita, Hua & 1997 & $4620 \cdots 7180$ & 0.5 & 0.7 & 6 & PN & & $\mathrm{CCD}$ & abs \\
\hline Echevarría et al. & 1989 & $3300-5600$ & 96 & 9 & 15 & $\mathrm{CV}$ & & Sp.Sc. & abs \\
\hline Fan et al. & 2000 & $5800-9800$ & $6.2,7.1$ & 13,14 & 8 & M7-L8 & $\mathrm{V}$ & DIS & rel \\
\hline Faÿ et al. & 1974 & $5000-7000$ & & 300 & 37 & $\mathrm{C}$ & & Sp.Sc. & counts \\
\hline Groppi, Hanson & 1986 & $6250-7150$ & & 1.3 & 9 & O4-O9 pec & $\mathrm{V}$ & $\mathrm{CCD}$ & norm \\
\hline Huenemoerder et al. & 1984 & $3600-5800$ & & 8,10 & 10 & FHB & & Reticon & rel \\
\hline Jüttner et al. & 1989 & $3900-4900$ & & 20000,25000 & 5 & $\mathrm{~B}$ & $\mathrm{~V}$ & $\mathrm{CCD}$ & norm \\
\hline Kaiser & 1987 & $3200-8500$ & & 10 & 26 & $\mathrm{Be}$ & III-V & Sp.Sc. & rel \\
\hline Kilian et al. & 1991 & $4060-5060$ & & 0.2 & 3 & B0-B2 & III, V & & norm \\
\hline Kirkpatrick et al. & 1991 & $6300-9000$ & & 18 & 77 & K5-M9 & $\mathrm{V}$ & $\mathrm{CCD}$ & rel \\
\hline Kolev, Tomov & 1993 & $3610-4930$ & & 0.35 & 1 & MWC 560 & & plate & norm \\
\hline Lennon et al. & 1992 & $3950-4950$ & 0.4 & 0.8 & 46 & O9-B9 & I & $\mathrm{CCD}$ & norm \\
\hline Liu et al. & $1999 a$ & $3700-8500$ & 4.9 & $7-23$ & 27 & $\mathrm{CV}$ & & $\mathrm{CCD}$ & abs \\
\hline Liu et al. & 1999b & $3800-8500$ & 4.9 & $12-33$ & 30 & $\mathrm{CV}$ & & $\mathrm{CCD}$ & abs \\
\hline Liu, $\mathrm{Hu}$ & 2000 & $3800-8500$ & 4.9 & $12-20$ & 32 & $\mathrm{CV}$ & & $\mathrm{CCD}$ & abs \\
\hline Lündstrom, Stenholm & 1984 & $4400-5900$ & 43 & 2.5 & 14 & WC, WN & & plate & counts \\
\hline Markova & 1994 & $3555-4800$ & 9 & 0.18 & 1 & P Cyg (B1-1.5) & Ia & plate & norm \\
\hline Markova, Zamanov & 1995 & $4840-6760$ & 18 & 0.36 & 1 & P Cyg (B1-1.5) & Ia & plate & norm \\
\hline Martin et al. & 1996 & $6400-8800$ & & 5.8 & 11 & $\mathrm{M}$ & $\mathrm{V}$ & $\mathrm{CCD}$ & rel \\
\hline Matheson et al. & 2001 & $3100-10500$ & & $3-15$ & 28 & SN & Ib-c & $\mathrm{CCD}$ & rel \\
\hline Moehler et al. & 1990 & $4000-5000$ & 60 & 2.5 & 92 & $\mathrm{O}-\mathrm{A}$ & $\mathrm{sd}$ & $\mathrm{CCD}$ & rel \\
\hline Oudmaijer & 1998 & $3850-10000$ & & 30000 & 1 & IRC+10420 (F8) & $\mathrm{Ia}$ & $\mathrm{CCD}$ & norm \\
\hline Parker et al. & 2001 & $3910-4740$ & 1.3 & 2.7 & 9 & $\mathrm{O}$ & I, II, V & $\mathrm{CCD}$ & norm \\
\hline Parthasarathy et al. & 2000 & $4350 \div 8820$ & & 0.3 & 1 & SAO 85766 (B1pe) & $\mathrm{I}$ & $\mathrm{CCD}$ & norm \\
\hline Pereira et al. & 2001 & $3100-7500$ & & $1.9,4.6$ & 25 & Be, T Tau & & $\mathrm{CCD}$ & rel \\
\hline Pritchet, van den Bergh & 1977 & $3800-6800$ & & $12 \div 45$ & 96 & O7-M9 & III-V & FTS & abs \\
\hline Schild et al. & 1990 & $4000-8000$ & & 7,14 & 12 & WR & & $\mathrm{CCD}$ & rel \\
\hline Stahl et al. & 1985 & $4600-4900$ & 20 & & 24 & A-B, pec & & plate & norm \\
\hline Stahl et al. & 1993 & $4050-9050$ & & 12000 & 1 & P Cyg & & $\mathrm{CCD}$ & norm \\
\hline Steele et al. & 1999 & $3670-5070$ & 0.5 & $0.67 \div 0.93$ & 20 & $\mathrm{Be}$ & III-V & $\mathrm{CCD}$ & norm \\
\hline Thévenin, Jasniewicz & 1992 & $4000-4700$ & 1.9 & 4.7 & 19 & A3-G0 & $\mathrm{I}$ & $\mathrm{CCD}$ & norm \\
\hline Torres-Dodgen & 1990 & $5800-8500$ & & 7 & 12 & O3-B3, pec & I, III & Reticon & norm \\
\hline Tull, Vogt & 1977 & $3900-6000$ & 4.4 & 0.28 & 1 & HD 88230 (M0) & $\mathrm{V}$ & Digicon & counts \\
\hline Vreux & 1983 & $5750-10350$ & 228 & 7.6 & 28 & WR & & Reticon & rel \\
\hline Vreux et al. & 1989 & $6150-10350$ & 228 & 7.6 & 12 & WR & & Reticon & rel \\
\hline $\mathrm{Xu}$ & 1991 & $\begin{array}{l}8160-8700 \\
4000-7850 \\
\end{array}$ & 33 & $\begin{array}{c}2 \\
12 \\
\end{array}$ & $\begin{array}{l}18 \\
14 \\
\end{array}$ & G0-M5 & III, V & $\begin{array}{l}\mathrm{CCD} \\
\mathrm{CCD} \\
\end{array}$ & $\begin{array}{c}\text { norm } \\
\text { abs }\end{array}$ \\
\hline
\end{tabular}


R. Sordo and U. Munari: The Asiago Database of Spectroscopic Databases (ADSD), Online Material p 9

Table 4. (continued)

\begin{tabular}{c|c|c|c|c|cc|c|}
\hline $\begin{array}{c}\text { Authors \& year } \\
\text { of publication }\end{array}$ & $\begin{array}{c}\text { Spectral } \\
\text { Range } \\
(\AA)\end{array}$ & $\begin{array}{c}\mathbf{D} \\
(\AA / \mathrm{pix}) \\
(\AA / \mathrm{mm})\end{array}$ & $\begin{array}{c}\mathbf{R} \text { o } \mathbf{R}_{P} \\
(\AA)\end{array}$ & $\begin{array}{c}\text { N. } \\
\text { Stars }\end{array}$ & $\begin{array}{c}\text { Spectrum } \\
\text { Lum. }\end{array}$ & $\begin{array}{c}\text { detector/ } \\
\text { instr. }\end{array}$ & $\begin{array}{c}\text { data } \\
\text { Class }\end{array}$ \\
\hline \hline
\end{tabular}

Ultraviolet libraries with data in graphical form

\begin{tabular}{|c|c|c|c|c|c|c|c|c|c|}
\hline Altamore et al. & 1992 & $\begin{array}{l}1190-3200 \\
3180-6500 \\
\end{array}$ & $12-15$ & $0.1-8$ & $\begin{array}{l}1 \\
1 \\
\end{array}$ & $\begin{array}{l}\text { KQ Pup (VV } \\
\text { KQ Pup (VV }\end{array}$ & & $\begin{array}{l}\text { IUE } \\
\text { plate }\end{array}$ & $\begin{array}{l}\text { abs } \\
\text { abs }\end{array}$ \\
\hline Artu et al. & 1989 & $1250-1986$ & & $? ?$ & 2 & B7, B9.5 & $\mathrm{V}$ & IUE & counts \\
\hline Bohlin et al. & 1990 & $1200-3200$ & & $5-6$ & 37 & $\mathrm{O}-\mathrm{G} 5, \mathrm{WD}$ & III-V & IUE & abs \\
\hline Carpenter et al. & 1988 & $1200-3230$ & & & 1 & $\gamma$ Cru (M3.4) & III & IUE & abs \\
\hline de Mello et al. & 2000 & $1200-1850$ & & & $10 \mathrm{~ms}$ & B0-B8 & $\mathrm{I}, \mathrm{III}, \mathrm{V}$ & IUE & norm \\
\hline Johnson & $1978 b$ & $920-3190$ & & $0.2,0.4$ & 3 & WR & & Sp.Sc. & abs \\
\hline Rountree, Sonneborn & 1991 & $1200-1900$ & & 0.25 & 14 & $\mathrm{~B}$ & III-V & IUE & norm \\
\hline Walborn, Bohlin & 1996 & $1000-1200$ & & 0.2 & 27 & OB & & Sp.Sc. & counts \\
\hline Walborn, Panek & 1984 & $1220 \cdots 1760$ & & 0.25 & 12 & O3-B1, pec & $\mathrm{V}$ & IUE & norm \\
\hline Wing et al. & 1983 & $2500-3200$ & & 10000 & 13 & G2-M3 & $\mathrm{I}-\mathrm{V}$ & IUE & abs \\
\hline
\end{tabular}

\section{Infrared libraries with data in graphical form}

\begin{tabular}{|c|c|c|c|c|c|c|c|c|}
\hline Ali et al. & 1995 & $2.15-2.36$ & & 1380 & 34 & $\mathrm{~F} 3-\mathrm{M} 6$ & $\mathrm{~V}$ & norm \\
\hline Clark, Steele & 2000 & $2.05-2.22$ & & $4.8 \div 5.2$ & 66 & $\mathrm{Be}$ & III-V & norm \\
\hline Conti, Howarth & 1999 & $0.98-1.1$ & & 3400,5600 & 70 & $\mathrm{O} 3-\mathrm{A} 5$, pec & I, III, V & norm \\
\hline Dhillon et al. & 2000 & $1.8-2.4$ & & $21-28$ & 25 & $\mathrm{~K} 2-\mathrm{M} 6, \mathrm{CV}$ & $\mathrm{V}$ & rel \\
\hline Eenens et al. & 1991 & $1-3.4$ & & $300-600$ & 8 & WC5-9 & & rel \\
\hline Figer et al. & 1997 & $2-2.4$ & & 525 & 38 & WR & & rel \\
\hline Forbes et al. & 1970 & $1.35-4.1$ & & 100 & 26 & G8-M10, pec & I-III & counts \\
\hline Genzel et al. & 2000 & $2.02-2.2$ & & 2000 & 18 & pec & & rel \\
\hline Hanson et al. & 1998 & $1.66-1.72$ & 3.5 & 8.7 & 34 & O7-B9, pec & $\mathrm{I}, \mathrm{V}$ & norm \\
\hline Howarth, Schmutz & 1992 & $0.97-1.12$ & 1.6 & $3-4$ & 24 & WR & & norm \\
\hline Ishii et al. & 2001 & $2-2.33$ & & 500 & 32 & YSO & & abs \\
\hline Johnson et al. & 1973 & $1.49 \div 2.5$ & & $0.5 \mathrm{~cm}^{-1}$ & 1 & $\chi \operatorname{Cyg}(\mathrm{S})$ & & counts \\
\hline Leggett et al. & 2000 & $0.75-2.5$ & & $18-62$ & 3 & $\mathrm{~T}$ & $\mathrm{~V}$ & rel \\
\hline Leggett et al. & 2001 & $1-2.55$ & & 600 & 7 & M6-L7 & $\mathrm{V}$ & rel \\
\hline Littlefair et al. & 2000 & $1.02-1.34$ & & $10 \div 13$ & 7 & $\mathrm{CV}$ & & rel \\
\hline Morris et al. & 1996 & $2.03-2.37$ & & 550 & 26 & $\mathrm{O}-\mathrm{B}, \mathrm{WN}, \mathrm{pec}$ & $\mathrm{I}, \mathrm{V}$ & norm \\
\hline Origlia et al. & 1993 & $1.51-1.72$ & & 1500 & 22 & A0-M5 & I,III,V & norm \\
\hline Ramirez et al. & 1997 & $2.19-2.34$ & & 1380 & 28 & K0-M6 & III & norm \\
\hline Reid et al. & 2001 & $1-2.5$ & & $18-82$ & 19 & M7-L8 & $\mathrm{V}$ & rel \\
\hline Ridgway et al. & 1984 & $3.6-4.1$ & & & 5 & $\mathrm{~K}, \mathrm{M}, \mathrm{C}, \mathrm{S}$ & & norm \\
\hline Rinehart et al. & 2002 & $7.5-14$ & & 600 & 15 & $\mathrm{PN}$ & & abs \\
\hline Smith, Houck & 2001 & $8-13$ & & 600 & 29 & $\mathrm{WN}, \mathrm{WC}$ & & abs \\
\hline Smith, Hummer & 1988 & $1.4-2.55$ & & 100 & 17 & $\mathrm{WC}$ & & counts \\
\hline Smith et al. & 2000 & $8-13$ & & $40-150$ & 13 & $\mathrm{YSO}$ & & abs \\
\hline Steele, Clark & 2001 & $1.53-1.69$ & & 5 & 57 & $\mathrm{Be}$ & III-V & norm \\
\hline Tamblyn et al. & 1996 & $2.04-2.22$ & & 2500 & 31 & $\mathrm{O} 6-\mathrm{B} 2, \mathrm{WN}$ & & norm \\
\hline Thompson et al. & 1973 & $1.4-2.5$ & & $0.5,1 \mathrm{~cm}^{-1}$ & 2 & $\mathrm{C}$ & & counts \\
\hline Volk et al. & 2000 & $2-43$ & & 400 & 5 & $\mathrm{C}$ & & abs \\
\hline
\end{tabular}

Combined libraries with data in graphical form

\begin{tabular}{ll|l|l|l|l|l|l}
\hline \hline Dessart et al. & 2000 & $0.1 \cdots 3.6$ & & & 3 & WC & abs \\
\hline
\end{tabular}

